\title{
TRAVELING-WAVE SOLUTIONS OF CONVECTION-DIFFUSION SYSTEMS BY CENTER MANIFOLD REDUCTION
}

\author{
STEPHEN SCHECTER
}

\begin{abstract}
Traveling waves $u(x-s t)$ for systems of conservation laws $u_{t}+D f(u) u_{x}=$ $\left(B(u) u_{x}\right)_{x}$ were studied by Majda and Pego (J. Differential Eqs. 56 (1985), 229-262) under the assumption that $u(-\infty)$ and $u(+\infty)$ are close. Their results were recently extended to general convection-diffusion systems $u_{t}+A(u) u_{x}=\left(B(u) u_{x}\right)_{x}$ by Sainsaulieu (SIAM $J$. Math. Anal. 27 (1996), 1286-1310). Sainsaulieu's proofs use fixed-point arguments in a function space. We rederive Sainsaulieu's results by center manifold reduction.
\end{abstract}

\section{IntRoduction}

A convection-diffusion system in one space dimension is a partial differential equation of the form

$$
u_{t}+A(u) u_{x}=\left(B(u) u_{x}\right)_{x} .
$$

Here $u \in \mathbb{R}^{n}$, and $A(u)$ and $B(u)$ are $n \times n$ matrices that we shall assume are $C^{2}$ functions of $u$. If we ignore diffusion, we have the convection system

$$
u_{t}+A(u) u_{x}=0 .
$$

If $A(u)=D f(u)$ for some flux function $f: \mathbb{R}^{n} \rightarrow \mathbb{R}^{n}$, then Eq. (1.2) becomes

$$
u_{t}+f(u)_{x}=0,
$$

a system of conservation laws, while Eq. (1.1) becomes

$$
u_{t}+f(u)_{x}=\left(B(u) u_{x}\right)_{x},
$$

a system of viscous conservation laws. The conservation law case occurs more often in applications and is far better studied; see [4] and [12]. However, equations of the form (1.2) and (1.1) that are not in conservation form arise in models of two-phase flow [13,10], deformation of elastic-plastic solids [14], and other applications.

One is forced to consider weak solutions of Eq. (1.3) or Eq. (1.2), since their solutions can become discontinuous even for analytic initial data. For a system of conservation laws (1.3), weak solutions are easily defined. If

$$
u(x)= \begin{cases}u_{-} & \text {for } x<0 \\ u_{+} & \text {for } x>0\end{cases}
$$

Date: January 21, 1998.

1991 Mathematics Subject Classification. 35K55, 35L67, 34C23.

Key words and phrases. convection-diffusion systems, nonconservation form, traveling waves, shock waves, center manifold reduction.

This work was supported in part by the National Science Foundation under Grant DMS-9501255, and by the Institute for Mathematics and its Applications with funds provided by the National Science Foundation. 
is a step function, then in the sense of distributions, $f(u)_{x}$ is the measure

$$
\left(f\left(u_{+}\right)-f\left(u_{-}\right)\right) \delta(x) .
$$

This leads to the Rankine-Hugoniot condition: A step function whose discontinuity propagates with speed $s$,

$$
u(x, t)= \begin{cases}u_{-} & \text {for } x<s t, \\ u_{+} & \text {for } x>s t,\end{cases}
$$

is a weak solution of Eq. (1.3) provided

$$
f\left(u_{+}\right)-f\left(u_{-}\right)-s\left(u_{+}-u_{-}\right)=0 .
$$

A step function (1.7) that satisfies Eq. (1.8), and is thus a weak solution of Eq. (1.3), is called a shock wave.

Unfortunately, the notion of a weak solution of a system of conservation laws is too generous; in particular, too many step function (1.7) satisfy the Rankine-Hugoniot condition (1.8), so that initial value problems with discontinuous initial data can have multiple solutions. The most successful remedy that has been proposed appears to be the viscous profile criterion. The idea is that a system in the form of Eq. (1.3) typically arises by assuming that the viscous term in a system in the form of Eq. (1.4) is small, and then setting it to zero.

According to the viscous profile criterion, a step function (1.7) is to be regarded as a solution of Eq. (1.3) provided the viscous system (1.4) has a traveling-wave solution $u(x-s t)$ with $u( \pm \infty)=u_{ \pm}, u^{\prime}( \pm \infty)=0$. If such a traveling wave exists, one easily checks that $u((x-s t) / \eta)$ is a traveling-wave solution of

$$
u_{t}+f(u)_{x}=\eta\left(B(u) u_{x}\right)_{x}
$$

As $\eta \rightarrow 0, u((x-s t) / \eta)$ converges to the discontinuous function (1.7). The viscous profile criterion thus accords with our intuition that solutions of Eq. (1.3) should be limits, as the viscosity coefficient $\eta$ approaches 0, of solutions of Eq. (1.9).

A traveling-wave solution $u(x-s t)$ of Eq. (1.4) satisfies the ordinary differential equation

$$
(D f(u)-s I) u^{\prime}=\left(B(u) u^{\prime}\right)^{\prime}
$$

Integrating and using the boundary conditions $u(-\infty)=u_{-}, u^{\prime}(-\infty)=0$, yields

$$
B(u) u^{\prime}=f(u)-f\left(u_{-}\right)-s\left(u-u_{-}\right) .
$$

Assuming $B(u)$ is invertible in a region of interest, we have

$$
u^{\prime}=B(u)^{-1}\left\{f(u)-f\left(u_{-}\right)-s\left(u-u_{-}\right)\right\},
$$

an ordinary differential equation with parameters $\left(u_{-}, s\right)$ and an equilibrium at $u=u_{-}$. The traveling wave $u(x-s t)$ corresponds to a solution $u(\xi)$ of Eq. (1.12) that goes from the equilibrium $u_{-}$at $\xi=-\infty$ to a second equilibrium $u_{+}$at $\xi=+\infty$. Since $u_{+}$must be an equilibrium of Eq. (1.12), we recover the Rankine-Hugoniot condition (1.8).

For further insight we consider the bifurcation diagram of Eq. (1.12) with $u_{-}$fixed and $s$ the parameter. Let us assume that $f$ is strictly hyperbolic at $u_{-}$, i.e., $D f\left(u_{-}\right)$has $n$ distinct real eigenvalues $\lambda_{1}<\ldots<\lambda_{n}$, with corresponding right eigenvectors $r_{1}, \ldots, r_{n}$. 
Under certain assumptions on $B\left(u_{-}\right)$(the Majda-Pego conditions) and $D^{2} f\left(u_{-}\right)$(genuine nonlinearity), Majda and Pego showed in [9] that the extended system

$$
\begin{aligned}
& u^{\prime}=B(u)^{-1}\left\{f(u)-f\left(u_{-}\right)-s\left(u-u_{-}\right)\right\}, \\
& s^{\prime}=0,
\end{aligned}
$$

has a two-dimensional center manifold at each $(u, s)=\left(u_{-}, \lambda_{i}\right)$. The flow of Eqs. (1.13)(1.14) on this center manifold exhibits a transcritical bifurcation as pictured in Figure 1.1. Thus for each $i=1, \ldots, n$, there emanates from $u_{-}$a one-sided curve of states $u_{+}(s), s<\lambda_{i}$, such that for each $s$ in the domain of $u_{+}(s)$ there is a solution $u(\xi)$ of Eq. (1.12) going from $u_{-}$to $u_{+}(s)$. Moreover, if we write $s=\lambda_{i}+\sigma$, then

$$
u_{+}(s)=u_{-}+k \sigma r_{i}+o(\sigma)
$$

for a certain nonzero constant $k$. We shall refer to this result, Theorem 3.1 of [9], as the Majda-Pego Theorem. The equilibria $u_{+}$in Figure 1.1 with $s>\lambda_{i}$ satisfy the RankineHugoniot condition but not the viscous profile criterion.

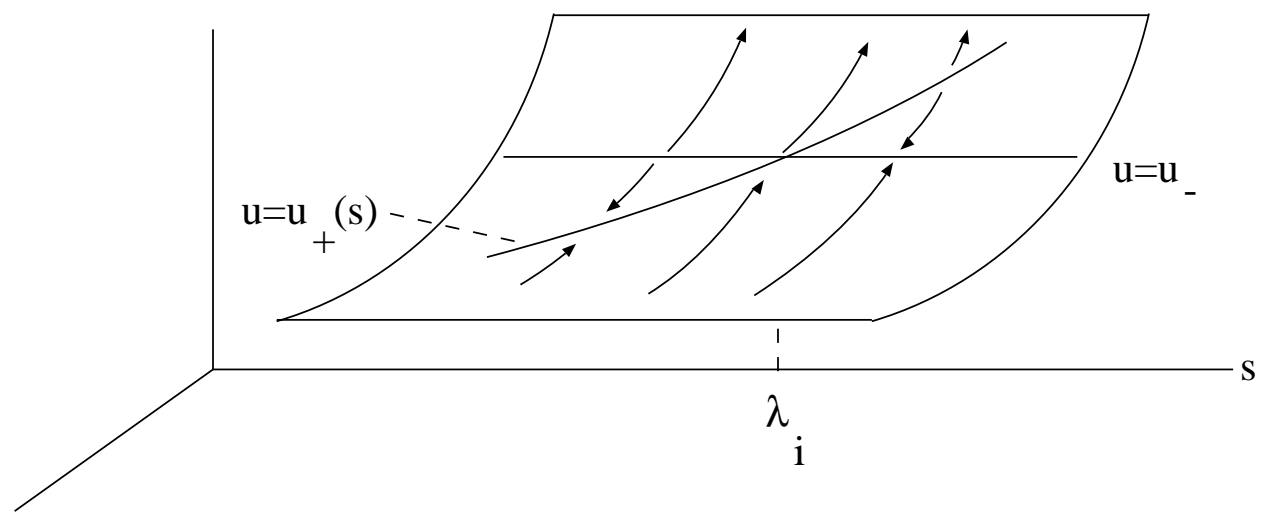

Figure 1.1. Geometry of the Majda-Pego Theorem.

The curves $u_{+}(s)$ for $s$ close to $\lambda_{i}$ are independent of the diffusion matrix $B(u)$, provided $B(u)$ satisfies appropriate conditions, and are in fact the right states of shock waves (1.7) that satisfy the Lax admissibility criterion [4]. However, Majda and Pego also show that other $B(u)$ will reverse the flow in Figure 1.1, causing the opposite half-curve of states $u_{+}$to become admissible. There are also other circumstances in which the shock waves admissible under the viscous profile criterion depend heavily on $B(u)$ [1].

If Eq. (1.2) is not in conservation form, it is not clear how to define weak solutions. The basic problem is how to view $A(u) u_{x}$ as a measure when $u(x)$ is given by (1.5); apparently we must multiply a step fuction by a delta function. In [2], Dal Maso, LeFloch, and Murat proposed a neat solution whose power is demonstrated in [7] and [8]. We first define a fixed family of paths connecting states in $\mathbb{R}^{n}, \phi:[0,1] \times \mathbb{R}^{n} \times \mathbb{R}^{n} \rightarrow \mathbb{R}^{n}, \phi\left(0, u_{-}, u_{+}\right)=u_{-}$, $\phi\left(1, u_{-}, u_{+}\right)=u_{+}$. Then, in the case of a step function (1.5), we associate with $A(u) u_{x}$ the measure $c \delta(x)$, where

$$
c=\int_{0}^{1} A(\phi(\tau)) \phi^{\prime}(\tau) d \tau
$$


and $\phi(\tau)=\phi\left(\tau, u_{-}, u_{+}\right)$. This leads to the DLM condition: A step function (1.7) is considered to be a weak solution of Eq. (1.2) provided

$$
\int_{0}^{1} A(\phi(\tau)) \phi^{\prime}(\tau) d \tau-s\left(u_{+}-u_{-}\right)=0
$$

Such a step function is again called a shock wave. Notice that if $A(u)=D f(u)$ for a flux function $f$, then $c=f\left(u_{+}\right)-f\left(u_{-}\right)$, consistent with (1.6). In this case the admissibility criterion (1.16) reduces to the Rankine-Hugoniot condition (1.8), independent of the choice of $\phi$. However, when Eq. (1.2) is not in conservation form, the admissibility criterion (1.16) depends on the choice of $\phi$.

In [6] LeFloch observed that shock waves (1.7) for Eq. (1.2), admissible under the DLM criterion, are limits of traveling-wave solutions of

$$
u_{t}+A(u) u_{x}=\eta\left(B(u) u_{x}\right)_{x}
$$

as $\eta \rightarrow 0$, provided the paths $\phi$ are chosen appropriately. Indeed, let $u(x-s t)$ be a travelingwave solution of Eq. (1.1) with $u( \pm \infty)=u_{ \pm}, u^{\prime}( \pm \infty)=0$. Then

$$
(A(u)-s I) u^{\prime}=\left(B(u) u^{\prime}\right)^{\prime} .
$$

Integration from $\xi=-\infty$ to $\xi=+\infty$ yields

$$
\int_{-\infty}^{+\infty} A(u(\xi)) u^{\prime}(\xi) d \xi-s\left(u_{+}-u_{-}\right)=0
$$

Now $u((x-s t) / \eta)$ is a traveling-wave solution of Eq. (1.17), which converges, as $\eta \rightarrow 0$, to the step function (1.7). Let $\psi:(0,1) \rightarrow \mathbb{R}$ be a bijection with $\psi^{\prime}>0$, and define $\phi(\tau)=\phi\left(\tau, u_{-}, u_{+}\right)$to be $u(\psi(\tau))$. Then (1.7) satisfies the DLM condition (1.16). Indeed, the integral in Eq. (1.16) is that in Eq. (1.19) after the change of variables $\xi=\psi(\tau)$.

LeFloch's observation leads to the question of what traveling waves exist for Eq. (1.1). Let us consider the analog of the strictly hyperbolic situation for conservation laws. Suppose that $A\left(u_{-}\right)$has $n$ distinct real eigenvalues $\lambda_{1}<\ldots<\lambda_{n}$, with right eigenvectors $r_{1}, \ldots, r_{n}$. Le Floch conjectured in [6] that under appropriate assumptions, the situation should be the same as that for conservation laws. In other words, for each $i=1, \ldots, n$ there should exist a one-sided curve $u_{+}(s), s<\lambda_{i}$, emanating from $u_{-}$parallel to the vector $r_{i}$, such that for each $s$, Eq. (1.1) has a traveling-wave solution $u(x-s t)$ going from $u_{-}$to $u_{+}(s)$.

Traveling-wave solutions of Eq. (1.1) are not as easily studied as those of Eq. (1.4) because the ordinary differential equation (1.18), unlike (1.10), cannot in general be integrated once. Moreover, the ODE (1.18), when converted to a first-order system on $\mathbb{R}^{2 n}$, has an $n$-dimensional plane of equilibria for each value of the parameter $s$, and is thus quite degenerate. Nevertheless LeFloch's conjecture was proved by Sainsaulieu in [11] using a fixed-point argument in a function space to find the connecting orbits. For invertible $B(u)$, Sainsaulieu's result is precisely analagous to the Majda-Pego Theorem. In addition, Sainsaulieu is able to treat certain degenerate diffusion matrices $B(u)$, which provides new information even in the conservation law case.

Our goal in this paper is to rederive Sainsaulieu's results using a more standard approach, center manifold reduction. Indeed, part of Sainsaulieu's argument is reminiscent of a proof of the center manifold theorem. The greater simplicity and geometric insight of the center manifold approach compensate, I hope, for the lack of novelty of the results.

The traveling-wave solutions of Eq. (1.1) that are found by Sainsaulieu's approach or ours stay near the left state $u_{-}$for all time, and have $u^{\prime}$ near zero for all time. In addition to 
finding curves of right states of such waves, Sainsaulieu addresses the question of whether there are others. We shall address this question only when $A(u)$ is strictly hyperbolic and $B(u) \equiv I$. Our argument, which is inspired by work of Peter Szmolyan on a different problem, emphasizes another aspect of the geometry of the situation.

The remainder of this paper is organized as follows. In Sec. 2 we find traveling waves for the viscous Burger's equation without integrating the traveling-wave equation. The geometry of our approach is clearest in this simple context, which does not require center manifold reduction. In Sec. 3 we review the center manifold theorem. In Sec. 4 we find traveling waves assuming $A(u)$ is strictly hyperbolic and $B(u) \equiv I$, which eliminates distracting algebra. In Sec. 5 we address uniqueness of the traveling waves in the same situation. In Sec. 6 we find traveling waves for general invertible $B(u)$, and in Sec. 7 for the degenerate diffusions considered by Sainsaulieu. Theorems are stated precisely at the beginning of Secs. 4, 5, 6, and 7. In Sec. 8 we make some concluding remarks about the statements of some results in [11].

\section{Viscous Burger's Equation}

Consider the viscous Burger's equation,

$$
u_{t}+u u_{x}=u_{x x}
$$

with $u \in \mathbb{R}$. We look for a traveling-wave solution $u(x-s t)$ with $u(-\infty)$ equal to a fixed state $u_{-}, u(+\infty)$ close to $u_{-}, u^{\prime}( \pm \infty)=0$, and $s$ close to $u_{-}$. Thus we write

$$
\begin{aligned}
& u=u(x-s t)=u_{-}+v(x-s t), \\
& s=u_{-}+\sigma,
\end{aligned}
$$

with

$$
\begin{array}{rlrl}
v(-\infty) & =0, & v^{\prime}(-\infty) & =0, \\
v(+\infty) & \sim 0, & v^{\prime}(+\infty)=0, \\
\sigma & \sim 0 .
\end{array}
$$

Substituting (2.2)-(2.3) into Eq. (2.1), we obtain

$$
-\left(u_{-}+\sigma\right) v^{\prime}+\left(u_{-}+v\right) v^{\prime}=v^{\prime \prime}
$$

which simplifies to

$$
(v-\sigma) v^{\prime}=v^{\prime \prime}
$$

Usually this equation is integrated once. However, to provide a model for our later work, we let $w=v^{\prime}$ and obtain the system

$$
\begin{aligned}
v^{\prime} & =w, \\
w^{\prime} & =(v-\sigma) w .
\end{aligned}
$$

This is an autonomous ordinary differential equation on $\mathbb{R}^{2}$ with a parameter $\sigma$ and, for each $\sigma$, the line of equilibria $w=0$. For $\sigma$ near 0 we wish to find solutions $(v(\xi), w(\xi))$ such that

$$
\begin{aligned}
& v(-\infty)=0, \quad w(-\infty)=0, \\
& v(+\infty) \sim 0, \quad w(+\infty)=0 .
\end{aligned}
$$


Invariant curves of the system (2.4)-(2.5) are easily found by dividing $w^{\prime}$ by $v^{\prime}$ and integrating:

$$
\begin{gathered}
\frac{d w}{d v}=\frac{w^{\prime}}{v^{\prime}}=\frac{(v-\sigma) w}{w}=v-\sigma ; \\
w=\frac{1}{2} v^{2}-\sigma v+w_{0} .
\end{gathered}
$$

To draw the phase portrait of the system (2.4)-(2.5) for fixed $\sigma$, we draw the curves $(2.6)$ in the $v w$-plane, indicate the equilibria on the line $w=0$, and draw arrows to denote the direction of flow. From (2.4), the flow is to the right in $w>0$ and to the left in $w<0$. See Figure 2.1.

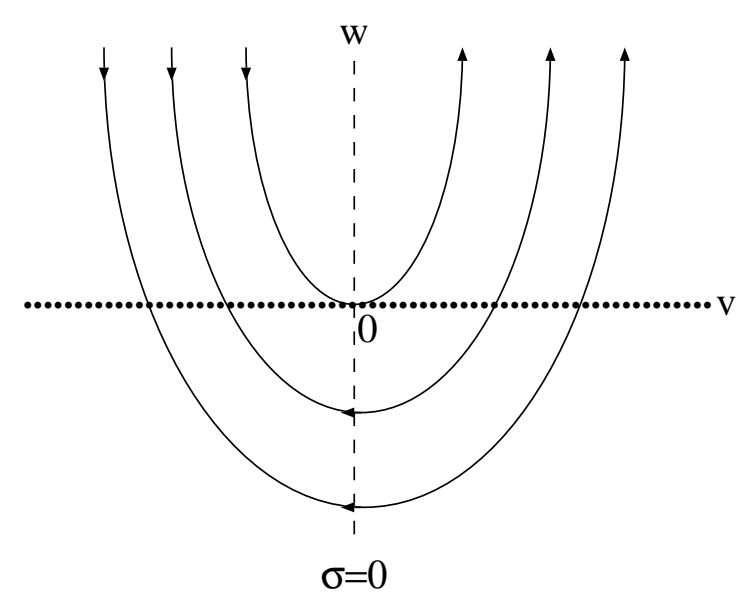

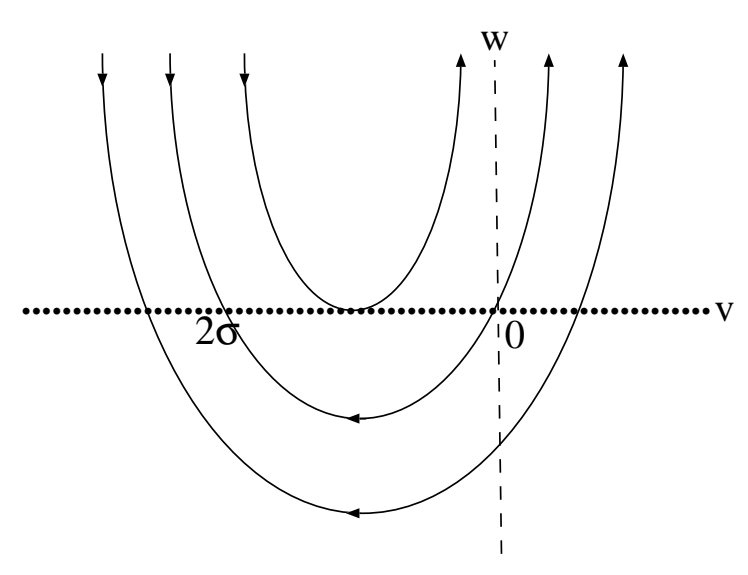

$\sigma<0$

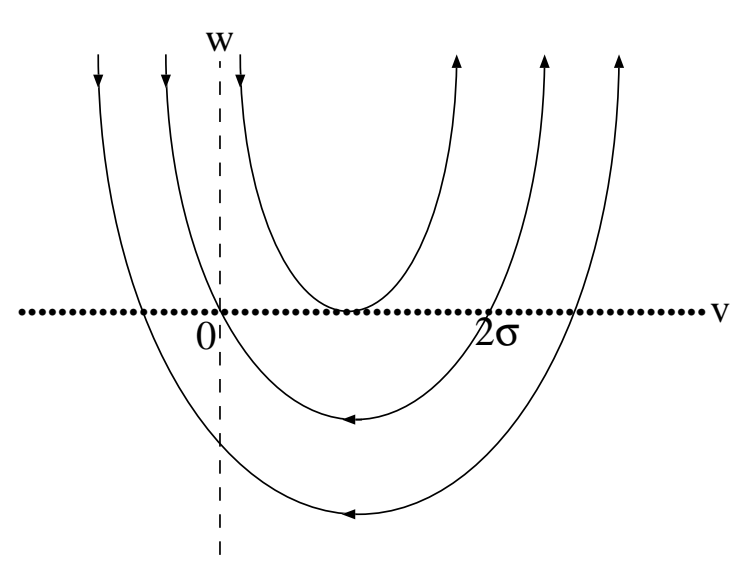

$\sigma>0$

Figure 2.1. Flow of the traveling-wave system (2.4)-(2.5) for Burger's equation.

From the pictures we see that for each $\sigma<0$ there is a solution as desired. More algebraically, to find $v(+\infty)$ we note that the curve $(2.6)$ that passes through $(v, w)=(0,0)$ 
is

$$
w=\frac{1}{2} v^{2}-\sigma v=v\left(\frac{1}{2} v-\sigma\right) .
$$

This curve reintersects the line of equilibria $w=0$ at $v=2 \sigma$. Thus $v(+\infty)=2 \sigma$, and only for $\sigma<0$ does the flow go from the equlibrium $(0,0)$ to the equilibrium $(2 \sigma, 0)$.

Finally, we note that for $s=u_{-}+\sigma, \sigma<0$, we have

$$
u_{+}=u_{-}+v(+\infty)=u_{-}+2 \sigma .
$$

This is a "curve" $u_{+}(s)$ of right states of traveling waves with left state $u_{-}$and speed $s$.

In this simple example the construction works for all $\sigma<0$, not just small $\sigma$.

\section{Center Manifold Theorem}

Consider an ordinary differential equation $z^{\prime}=f(z)$ on $\mathbb{R}^{q}$ with $f(0)=0$. Assume that $f$ is $C^{p}$ with $p \geq 1$. The center subspace of $D f(0)$ is the subspace of $\mathbb{R}^{q}$ spanned by all eigenvectors and generalized eigenvectors of $D f(0)$ corresponding to eigenvalues with real part zero. The Center Manifold Theorem states that the system $z^{\prime}=f(z)$ has a locally invariant $C^{p}$ manifold through 0 that is tangent at 0 to the center subspace of $D f(0)$. Moreover, any solution of $z^{\prime}=f(z)$ that stays near the origin for $-\infty<t<+\infty$ lies in this manifold, which is called a center manifold for the system.

Suppose a linear change of coordinates puts $z^{\prime}=f(z)$ into the triangular form

$$
\begin{aligned}
x^{\prime} & =M x+P y+m(x, y), \\
y^{\prime} & =N y+n(x, y),
\end{aligned}
$$

where $m$ and $n$ are $C^{p}$ functions that are $o(\|(x, y)\|)$. Suppose, moreover, that the spectrum of $M$ is contained in $\{\lambda: \operatorname{Re} \lambda=0\}$, and the spectrum of $N$ is contained in $\{\lambda: \operatorname{Re} \lambda \neq 0\}$. Then the center subspace of the linearization of this system at the origin is $x$-space. Hence, according to the Center Manifold Theorem, there is a $C^{p}$ function $y=g(x)$, defined on a neighborhood of 0 in $x$-space, with $g(0)=0$ and $D g(0)=0$, such that the manifold $\{(x, y): y=g(x)\}$ is a center manifold for the system (3.1)-(3.2). The differential equation (3.1)-(3.2) restricted to this manifold is the $C^{p}$ system

$$
x^{\prime}=M x+P g(x)+m(x, g(x)) .
$$

The Center Manifold Theorem can be applied to a differential equation with parameters $z^{\prime}=f(z, \mu)$ if one first appends the system $\mu^{\prime}=0$.

Early proofs of the Center Manifold Theorem only showed that center manifolds are $C^{p-1}$. For a modern proof that shows they are $C^{p}$, see [15]. For examples of the use of the Center Manifold Theorem in concrete problems, see [3].

\section{IDEntity Diffusion Matrix}

In this section we consider the convection-diffusion system (1.1) with $A(u)$ strictly hyperbolic and $B(u) \equiv I$. Thus we consider the system

$$
u_{t}+A(u) u_{x}=u_{x x} .
$$

Recall that the matrix $A\left(u_{-}\right)$is strictly hyperbolic if it has $n$ distinct real eigenvalues $\lambda_{1}<$ $\ldots<\lambda_{n}$. Let $\ell_{1}, \ldots, \ell_{n}$ and $r_{1}, \ldots, r_{n}$ denote corresponding left and right eigenvectors, chosen so that $\ell_{i} r_{i}=1$ for $i=1, \ldots, n$. The $j$ th characteristic field is genuinely nonlinear 
at $u_{-}$if $\ell_{j}\left(D A\left(u_{-}\right) r_{j}\right) r_{j} \neq 0$. After replacing $r_{j}$ by $-r_{j}$ and $\ell_{j}$ by $-\ell_{j}$ if necessary, we may assume that $\ell_{j}\left(D A\left(u_{-}\right) r_{j}\right) r_{j}>0$.

Theorem 4.1. In Eq. (4.1) assume:

(1) $A(u)$ is a $C^{2}$ function of $u$.

(2) $A\left(u_{-}\right)$is strictly hyperbolic.

(3) The jth characteristic field is genuinely nonlinear at $u_{-}$.

Let $a_{j}=\ell_{j}\left(D A\left(u_{-}\right) r_{j}\right) r_{j}>0$. Then for small $\sigma<0$ there exists a traveling-wave solution $u(x-s t)$ of Eq. (4.1), s $=\lambda_{j}+\sigma$, with

$$
\begin{aligned}
u(-\infty) & =u_{-}, \\
u^{\prime}(-\infty) & =0, \\
u(+\infty) & =u_{-}+\frac{2}{a_{j}} \sigma r_{j}+o(\sigma), \\
u^{\prime}(+\infty) & =0,
\end{aligned}
$$

and

$$
u(\xi) \sim u_{-} \text {and } u^{\prime}(\xi) \sim 0 \text { for }-\infty<\xi<+\infty .
$$

The formula for $u(+\infty)$ gives a curve $u_{+}(s)$ of right states of traveling waves with left state $u_{-}$and speed $s$.

Proof. We shall do the case $j=1$. Let

$$
\begin{aligned}
& u=u(x-s t)=u_{-}+v(x-s t), \\
& s=\lambda_{1}+\sigma,
\end{aligned}
$$

with

$$
\begin{array}{rlrl}
v(-\infty) & =0, & v^{\prime}(-\infty) & =0, \\
v(+\infty) & \sim 0, & v^{\prime}(+\infty)=0, \\
\sigma & \sim 0,
\end{array}
$$

and

$$
v(\xi) \sim 0 \text { and } v^{\prime}(\xi) \sim 0 \text { for }-\infty<\xi<+\infty .
$$

Substituting (4.2)-(4.3) into Eq. (4.1), we obtain

$$
\left(A\left(u_{-}+v\right)-\left(\lambda_{1}+\sigma\right) I\right) v^{\prime}=v^{\prime \prime} .
$$

Let $C(v)=A\left(u_{-}+v\right)-\lambda_{1} I$. Then Eq. (4.8) becomes

$$
(C(v)-\sigma I) v^{\prime}=v^{\prime \prime},
$$

where:

$\left(1^{\prime}\right) C(v)$ is a $C^{2}$ function of $v$.

$\left(2^{\prime}\right) C(0)$ has distinct real eigenvalues $0, \lambda_{2}-\lambda_{1}, \ldots, \lambda_{n}-\lambda_{1}$, with corresponding left and right eigenvectors $\ell_{1}, \ldots, \ell_{n}$ and $r_{1}, \ldots, r_{n}$.

$\left(3^{\prime}\right) \ell_{1}\left(D C(0) r_{1}\right) r_{1}=a_{1}>0$. 
In Eq. (4.9) let $w=v^{\prime}$. We obtain the system

$$
\begin{aligned}
v^{\prime} & =w, \\
w^{\prime} & =(C(v)-\sigma I) w .
\end{aligned}
$$

Since $\sigma$ is a parameter and we wish to use center manifold reduction, we append the equation

$$
\sigma^{\prime}=0 \text {. }
$$

System (4.10)-(4.12) is a $C^{2}$ autonomous ODE on $\mathbb{R}^{2 n+1}$ with the $(n+1)$-dimensional plane of equilibria $w=0$. For $\sigma$ near 0 we want to find solutions $(v(\xi), w(\xi), \sigma)$ such that

$$
\begin{aligned}
& v(-\infty)=0, \quad w(-\infty)=0, \\
& v(+\infty) \sim 0, \quad w(+\infty)=0,
\end{aligned}
$$

and

$$
v(\xi) \sim 0 \text { and } w(\xi) \sim 0 \text { for }-\infty<\xi<+\infty .
$$

Such solutions must lie in the center manifold of $(v, w, \sigma)=(0,0,0)$.

Let $R=\left(r_{1}, \ldots, r_{n}\right)$, an $n \times n$ matrix, so that

$$
R^{-1}=\left(\begin{array}{c}
\ell_{1} \\
\vdots \\
\ell_{n}
\end{array}\right) \text {. }
$$

Make the change of variables $v=R x$ and $w=R y$. Then the system (4.10)-(4.12) becomes the $C^{2}$ system

$$
\begin{aligned}
x^{\prime} & =y, \\
y^{\prime} & =R^{-1}(C(R x)-\sigma I) R y, \\
\sigma^{\prime} & =0 .
\end{aligned}
$$

The linearization of the system (4.17)-(4.19) at the equilibrium $(x, y, \sigma)=(0,0,0)$ is

$$
\begin{aligned}
x^{\prime} & =y, \\
y^{\prime} & =R^{-1} C(0) R y, \\
\sigma^{\prime} & =0 .
\end{aligned}
$$

Notice that $R^{-1} C(0) R$ is a diagonal matrix with entries $0, \lambda_{2}-\lambda_{1}, \ldots, \lambda_{n}-\lambda_{1}$.

The characteristic polynomial of the linear system (4.20)-(4.22) has 0 as a root of multiplicity $n+2$ and has no other roots on the imaginary axis. The $(n+2)$-dimensional generalized eigenspace for the eigenvalue 0 is $x y_{1} \sigma$-space. The center manifold is therefore tangent to this space, so its equations are

$$
y_{i}=g_{i}\left(x, y_{1}, \sigma\right), \quad i=2, \ldots, n,
$$

with each $g_{i}$ a $C^{2}$ function, $g_{i}(0,0,0)=0$, and $D g_{i}(0,0,0)=0$. However, all points $(x, 0, \sigma)$ are equilibria, hence are in the center manifold. Therefore we must have $g_{i}(x, 0, \sigma) \equiv 0$ for $i=2, \ldots, n$. Hence

$$
y_{i}=y_{1} h_{i}\left(x, y_{1}, \sigma\right), \quad i=2, \ldots, n,
$$

with each $h_{i}$ a $C^{1}$ function such that $y_{1} h_{i}$ is $C^{2}$, and $h_{i}(0,0,0)=0$ because $D g_{i}(0,0,0)=0$. 
The differential equation (4.17)-(4.19), restricted to the center manifold, becomes

$$
\begin{aligned}
x_{1}^{\prime} & =y_{1}, \\
x_{2}^{\prime} & =y_{1} h_{2}, \\
\vdots & \\
x_{n}^{\prime} & =y_{1} h_{n}, \\
y_{1}^{\prime} & =\ell_{1}(C(R x)-\sigma I) R\left(y_{1}, y_{1} h_{2}, \ldots, y_{1} h_{n}\right)^{\top}, \\
\sigma^{\prime} & =0 .
\end{aligned}
$$

Using $\ell_{1} C(0)=0$ and $h_{i}(0,0,0)=0$, Eq. (4.27) can be expanded as follows:

$$
\begin{aligned}
y_{1}^{\prime} & =y_{1} \ell_{1}(C(0)+D C(0) R x+\ldots-\sigma I) R\left(1, h_{2}, \ldots, h_{n}\right)^{\top} \\
& =y_{1} \ell_{1}\left\{(D C(0) R x-\sigma I) R(1,0, \ldots, 0)^{\top}+\ldots\right\} \\
& =y_{1} \ell_{1}\left\{\left(\sum_{i=1}^{n} x_{i} D C(0) r_{i}-\sigma I\right) r_{1}+\ldots\right\} \\
& =y_{1}\left(\sum_{i=1}^{n} a_{i} x_{i}-\sigma+\ldots\right),
\end{aligned}
$$

where $a_{i}=\ell_{1}\left(D C(0) r_{i}\right) r_{1}$, so $a_{1}>0$ by $\left(3^{\prime}\right)$, and " $+\ldots$ " indicates higher-order terms.

Eqs. (4.24)-(4.27) constitute a $C^{2}$ autonomous ODE on $\mathbb{R}^{n+1}$ with a parameter $\sigma$. For each $\sigma$ we have the $n$-dimensional plane of equlibria $y_{1}=0$. For $\sigma$ near 0 we wish to find solutions that go from the equilibrium $\left(x, y_{1}\right)=(0,0)$ to a nearby equilibrium $(x, 0)$.

To find invariant curves of the system (4.24)-(4.27), we first divide Eqs. (4.25)-(4.27) by Eq. (4.24):

$$
\begin{aligned}
\frac{d x_{2}}{d x_{1}} & =\frac{x_{2}^{\prime}}{x_{1}^{\prime}}=h_{2}, \\
\vdots & \\
\frac{d x_{n}}{d x_{1}} & =\frac{x_{n}^{\prime}}{x_{1}^{\prime}}=h_{n}, \\
\frac{d y_{1}}{d x_{1}} & =\frac{y_{1}^{\prime}}{x_{1}^{\prime}}=\sum_{i=1}^{n} a_{i} x_{i}-\sigma+\ldots .
\end{aligned}
$$

Eqs. (4.29)-(4.31) are a $C^{1}$ nonautonomous ODE on $\mathbb{R}^{n}$ with $x_{1}$ playing the role of time and $\sigma$ a parameter.

We are interested in solutions

$$
\left(x_{2}\left(x_{1}, \sigma\right), \ldots, x_{n}\left(x_{1}, \sigma\right), y_{1}\left(x_{1}, \sigma\right)\right)
$$

with

$$
\left(x_{2}(0, \sigma), \ldots, x_{n}(0, \sigma), y_{1}(0, \sigma)\right)=(0, \ldots, 0,0) .
$$

These solutions of course have the form

$$
\begin{gathered}
x_{2}=x_{1} \phi_{2}\left(x_{1}, \sigma\right), \\
\vdots \\
x_{n}=x_{1} \phi_{n}\left(x_{1}, \sigma\right), \\
y_{1}=x_{1} \psi\left(x_{1}, \sigma\right) .
\end{gathered}
$$


Here each $\phi_{i}$ and $\psi$ are $C^{1}$ by Lemma 4.2, which will be proved at the end of this section. Since $h_{i}(0,0,0)=0$ for $i=2, \ldots, n$, we have $\phi_{i}(0,0)=0$ for $i=2, \ldots, n$. Similarly, $\psi(0,0)=0$. We can easily calculate $\psi$ to first order by substituting Eq. (4.34) into Eq. (4.31). We obtain

$$
\psi\left(x_{1}, \sigma\right)=\frac{a_{1}}{2} x_{1}-\sigma+\ldots
$$

For each $\sigma$ the curve given by Eqs. (4.32)-(4.34) passes through $\left(x, y_{1}\right)=(0,0)$; that is how it was chosen. It again meets the plane of equilibria $y_{1}=0$ where $\psi=0$. Since $a_{1} \neq 0$ by assumption, we can solve the equation $\psi\left(x_{1}, \sigma\right)=0$ for $x_{1}$ near the origin by the Implicit Function Theorem:

$$
x_{1}=\frac{2}{a_{1}} \sigma+o(\sigma) \text {. }
$$

Since $a_{1}>0$, the curves $y_{1}=x_{1} \psi\left(x_{1}, \sigma\right)$ are as pictured in Figure 4.1. Since $x_{1}^{\prime}=y_{1}$, only for $\sigma<0$ does the connection go from the origin to the nearby equilibrium with $y_{1}=0$.

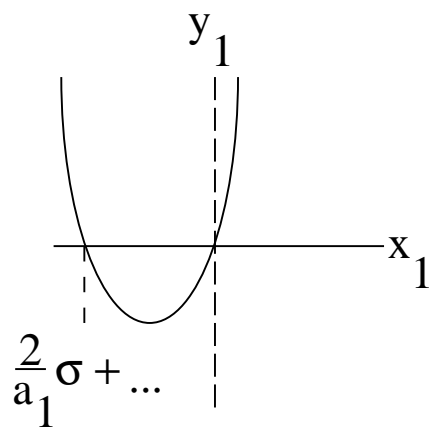

$\sigma<0$

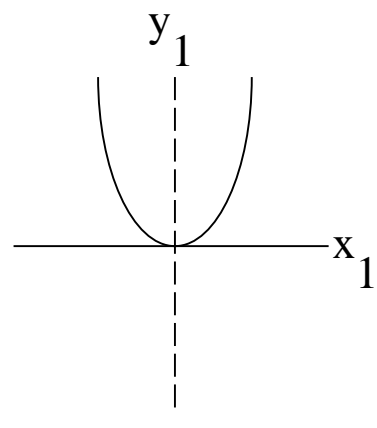

$\sigma=0$

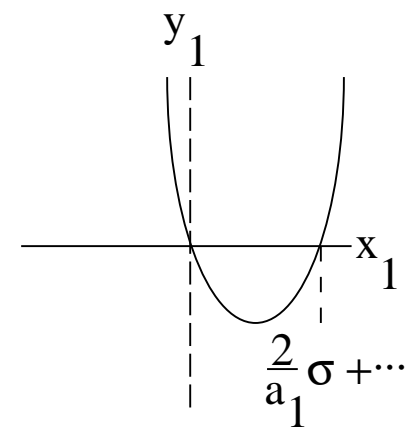

$\sigma>0$

Figure 4.1. The curves $y_{1}=x_{1} \psi\left(x_{1}, \sigma\right)$.

Substituting formula (4.36) into Eqs. (4.32)-(4.33) and recalling that $\phi_{i}(0,0)=0$ for $i=2, \ldots, n$, we obtain

$$
x_{i}=o(\sigma), \quad i=2, \ldots, n \text {. }
$$

Eqs. (4.36)-(4.37) yield the desired formula for $u(+\infty)$ after the change of variables $v=$ $R x$.

Lemma 4.2. Consider the nonautonomous parameterized family of ODEs $\frac{d x}{d t}=f(t, x, \mu)$, with $f: \mathbb{R} \times \mathbb{R}^{n} \times \mathbb{R}^{p} \rightarrow \mathbb{R}^{n}$ a $C^{1}$ function. Let $x=\alpha(t, \mu)$ be the family of solutions with $\alpha(0, \mu)=0$. Then $\alpha(t, \mu)=t \beta(t, \mu)$ with $\beta(t, \mu)$ a $C^{1}$ function. 
Proof. Since a $C^{1}$ ODE has a $C^{1}$ flow, $\alpha(t, \mu)$ is $C^{1}$. Moreover,

$$
\begin{aligned}
\alpha(t, \mu) & =\int_{0}^{t} f(\tau, \alpha(\tau, \mu), \mu) d \tau \\
& =\int_{0}^{1} f(t \theta, \alpha(t \theta, \mu), \mu) t d \theta \\
& =t \int_{0}^{1} f(t \theta, \alpha(t \theta, \mu), \mu) d \theta \\
& =t \beta(t, \mu),
\end{aligned}
$$

where $\beta(t, \mu)$ is $C^{1}$ because $f$ and $\alpha$ are.

\section{UNIQUENESS}

In Eq. (4.1), if $A(u)$ is a $C^{2}$ function of $u, A\left(u_{-}\right)$is strictly hyperbolic, and all $n$ characteristic fields are genuinely nonlinear at $u_{-}$, then Theorem 4.1 asserts the existence of $n$ one-sided curves $u_{i}(s), s<\lambda_{i}$, emanating from $u_{-}$. For each $s$ in the domain of $u_{i}(s)$, there is a traveling-wave solution $u(x-s t)$ for Eq. (4.1) such that

$$
\begin{array}{cc}
u(-\infty)=u_{-}, & u^{\prime}(-\infty)=0, \\
u(+\infty)=u_{i}(s), & u^{\prime}(+\infty)=0,
\end{array}
$$

and

$$
u(\xi) \sim u_{-} \text {and } u^{\prime}(\xi) \sim 0 \text { for }-\infty<\xi<+\infty .
$$

In this section we shall show

Theorem 5.1. Under the above assumptions, there is an $\epsilon>0$ such that if $u(x-s t)$ is a traveling-wave solution of Eq. (4.1) such that

$$
\begin{aligned}
u(-\infty)=u_{-}, & u^{\prime}(-\infty)=0 \\
\left\|u(+\infty)-u_{-}\right\|<\epsilon, & u^{\prime}(+\infty)=0
\end{aligned}
$$

and

$$
\left\|u(\xi)-u_{-}\right\|<\epsilon \text { and }\left\|u^{\prime}(\xi)\right\|<\epsilon \text { for }-\infty<\xi<+\infty,
$$

then there is a number $\xi_{0}$ such that $u\left(x-s t+\xi_{0}\right)$ is one of the traveling waves whose existence is given by Theorem 4.1.

Proof. We look for traveling-wave solutions $u(x-s t)$ of Eq. (4.1) satisfying (5.1)-(5.3). Then

$$
(A(u)-s I) u^{\prime}=u^{\prime \prime} .
$$

Letting $w=u^{\prime}$, we obtain the system

$$
\begin{aligned}
u^{\prime} & =w, \\
w^{\prime} & =(A(u)-s I) w .
\end{aligned}
$$

We append the equation

$$
s^{\prime}=0 \text {. }
$$

System (5.4)-(5.6) is a $C^{2}$ autonomous ODE on $\mathbb{R}^{2 n+1}$ with the $(n+1)$-dimensional plane of equilibria $\mathcal{P}=\{(u, w, s): w=0\}$. Each $2 n$-dimensional plane $s=$ constant is invariant. We must find solutions $(u(\xi), w(\xi), s)$ of $(5.4)-(5.6)$ such that 
(1) $\lim _{\xi \rightarrow-\infty}(u(\xi), w(\xi), s)=\left(u_{-}, 0, s\right)$,

(2) $\lim _{\xi \rightarrow+\infty}(u(\xi), w(\xi), s)=\left(u_{+}, 0, s\right)$ with $u_{+} \sim u_{-}$, and

(3) $u(\xi) \sim u_{-}$and $u^{\prime}(\xi) \sim 0$ for $-\infty<\xi<+\infty$.

Since $(u(\xi), w(\xi), s)$ is a solution of system (5.4)-(5.6) if and only if the shift $\left(u\left(\xi+\xi_{0}\right), w(\xi+\right.$ $\left.\left.\xi_{0}\right), s\right)$ is a solution, we shall ignore such shifts in the following.

Let $\mathcal{L}=\left\{(u, w, s): u=u_{-}\right.$and $\left.w=0\right\}$, a line in $\mathcal{P}$. Then according to (1)-(3) we are looking for solutions $(u(\xi), w(\xi), s)$ of $(5.4)-(5.6)$ that approach a point of $\mathcal{L}$ a $\xi \rightarrow-\infty$, that approach a point in $\mathcal{P}$ near $\mathcal{L}$ as $\xi \rightarrow+\infty$, and that stay near $\mathcal{L}$ for all time.

At any point $\left(u_{0}, 0, s_{0}\right) \in \mathcal{P}$, let $u=u_{0}+v$ and $s=s_{0}+\sigma$. Then the linearization of system $(5.4)-(5.6)$ at $\left(u_{0}, 0, s_{0}\right)$ is

$$
\begin{aligned}
v^{\prime} & =w, \\
w^{\prime} & =\left(A\left(u_{0}\right)-s_{0} I\right) w . \\
\sigma^{\prime} & =0 .
\end{aligned}
$$

This linear system has $v \sigma$-space as an invariant subspace on which it is identically 0 (corresponding to the plane of equilibria $\mathcal{P}$ ). If no eigenvalue of $A\left(u_{0}\right)-s_{0} I$ is 0 , then there is a complementary invariant subspace on which the eigenvalues are those of $A\left(u_{0}\right)-s_{0} I$.

According to Theorem 4.1, emanating from each point $\left(u_{-}, 0, \lambda_{i}\right) \in \mathcal{L}$ is a one-sided curve $\left(u_{i}(s), 0, s\right), s<\lambda_{i}$, in $\mathcal{P}$, such that for each $s$ in the domain of $u_{i}(s)$, there is a solution of the system $(5.4)-(5.6)$ that goes from $\left(u_{-}, 0, s\right)$ to $\left(u_{i}(s), 0, s\right)$ while staying near $\mathcal{L}$. Moreover, the proof actually shows that for sufficiently small $\delta>0$, all solutions $(u(\xi), w(\xi), s)$ of the system (5.4)-(5.6) that satisfy

(a) $\lim _{\xi \rightarrow-\infty}(u(\xi), w(\xi), s)=\left(u_{-}, 0, s\right)$,

(b) $\lim _{\xi \rightarrow+\infty}(u(\xi), w(\xi), s)=\left(u_{+}, 0, s\right)$ with $\left\|u_{+}-u_{-}\right\|<\delta$,

(c) $\left\|u(\xi)-u_{-}\right\|<\delta$ and $\|w(\xi)\|<\delta$ for $-\infty<\xi<+\infty$, and

(d) $\lambda_{i}-\delta<s<\lambda_{i}+\delta$ for some $i=1, \ldots, n$,

are given by the theorem.

Fix such a $\delta>0$. Let $\gamma>0$, and let

$$
\begin{aligned}
\mathcal{E}_{0} & =\left\{(u, 0, s):\left\|u-u_{-}\right\| \leq \gamma \text { and } s \leq \lambda_{1}-\delta\right\}, \\
\mathcal{E}_{1} & =\left\{(u, 0, s):\left\|u-u_{-}\right\| \leq \gamma \text { and } \lambda_{1}+\delta \leq s \leq \lambda_{2}-\delta\right\}, \\
& \vdots \\
\mathcal{E}_{n-1} & =\left\{(u, 0, s):\left\|u-u_{-}\right\| \leq \gamma \text { and } \lambda_{n-1}+\delta \leq s \leq \lambda_{n}-\delta\right\}, \\
\mathcal{E}_{n} & =\left\{(u, 0, s):\left\|u-u_{-}\right\| \leq \gamma \text { and } \lambda_{n}+\delta \leq s\right\},
\end{aligned}
$$

Each of $\mathcal{E}_{0}, \ldots, \mathcal{E}_{n}$ is a closed $n$-dimensional manifold-with-corners consisting entirely of equilibria; $\mathcal{E}_{1}, \ldots, \mathcal{E}_{n-1}$ are compact. We shall assume $\gamma$ is sufficently small so that each $\mathcal{E}_{i}$ is normally hyperbolic, which in this simple context just means that for each $(u, 0, s) \in \mathcal{E}_{i}$, $A(u)-s I$ has no eigenvalue with real part 0 . More precisely, at $(u, 0, s) \in \mathcal{E}_{i}, A(u)-s I$ has $n-i$ positive real eigenvalues and $i$ negative real eigenvalues.

Figure 5.1 may be helpful in keeping track of the notation and geometry of this section. It is supposed to show the case $n=2$, but $u$-space and $w$-space are drawn as if they were one-dimensional.

By results of Fenichel [5], each $\mathcal{E}_{i}, i=1, \ldots, n-1$ (the compact ones), has local stable and unstable manifolds that fiber over $\mathcal{E}_{i}$. This means: through each point $(u, 0, s)$ in $\mathcal{E}_{i}$ there 


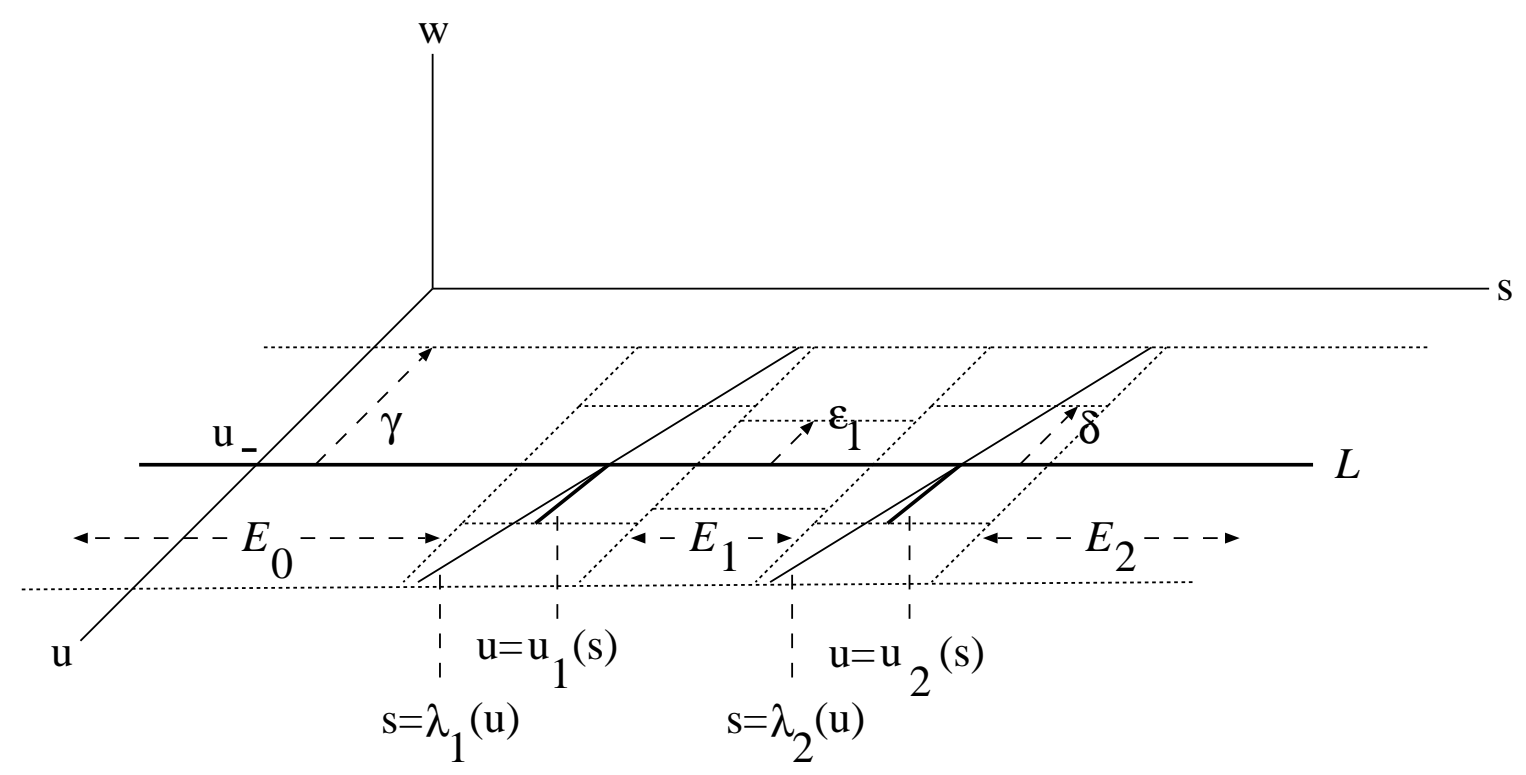

Figure 5.1. Notation and geometry in the case $n=2$ for the proof of the uniqueness theorem; $u$-space and $w$-space are drawn as if they were onedimensional. The eigenvalues of $A(u)$ are $\lambda_{1}(u)<\lambda_{2}(u)$. $\mathcal{E}_{0}$ is normally repelling, $\mathcal{E}_{1}$ is normally of saddle type, and $\mathcal{E}_{2}$ is normally attracting.

is a manifold $\mathcal{U}_{i}(u, 0, s)$, of dimension $n-i$, consisting of orbits asymptotic to $(u, 0, s)$ in backward time, and a manifold $\mathcal{S}_{i}$, of dimension $i$, consisting of orbits asymptotic to $(u, 0, s)$ in forward time; the $\mathcal{U}_{i}(u, 0, s)$ fit together to form a manifold $\mathcal{U}_{i}$ of dimension $2 n+1-i$, the local unstable manifold of $\mathcal{E}_{i}$, and the $\mathcal{S}_{i}(u, 0, s)$ fit together to form a manifold $\mathcal{S}_{i}$ of dimension $n+1+i$, the local stable manifold of $\mathcal{E}_{i} ; \mathcal{U}_{i}$ and $\mathcal{S}_{i}$ intersect only along $\mathcal{E}_{i}$; and there is a neighborhood $\mathcal{N}_{i}$ of $\mathcal{E}_{i}$ such that any orbit in $\mathcal{N}_{i}$ that is backward asymptotic to $\mathcal{E}_{i}$ without leaving $\mathcal{N}_{i}$ is contained in $\mathcal{U}_{i}$, and any orbit in $\mathcal{N}_{i}$ that is forward asymptotic to $\mathcal{E}_{i}$ without leaving $\mathcal{N}_{i}$ is contained in $\mathcal{S}_{i}$. Since $\mathcal{U}_{i}$ and $\mathcal{S}_{i}$ intersect only along $\mathcal{E}_{i}$, this implies that for each $\left(u_{-}, 0, s\right) \in \mathcal{E}_{i}$, there is no solution of the system (5.4)-(5.6) that aproaches $\left(u_{-}, 0, s\right)$ in backward time and approaches a point $(u, 0, s) \in \mathcal{E}_{i}$ in forward time while staying in $\mathcal{N}_{i}$. In particular, because of the compactness of $\mathcal{E}_{i}$, there exists $\epsilon_{i}>0$ such that there is no solution $(u(\xi), w(\xi), s)$ of the system (5.4)-(5.6) with $\lambda_{i}+\delta \leq s \leq \lambda_{i+1}-\delta$ that satisfies (a) $-(\mathrm{c})$ with $\delta$ replaced by $\epsilon_{i}$.

The set $\mathcal{E}_{0}$, on which the system $(5.4)-(5.6)$ is normally repelling, is not compact. Nevertheless, Fenichel's results imply the existence of a neighborhood of $\mathcal{E}_{0}$ in uws-space consisting entirely of a neighborhood of $\mathcal{E}_{0}$ in $\mathcal{P}$ and orbits that approach it in backward time. Thus there is no solution $(u(\xi), w(\xi), s)$ of the system (5.4)-(5.6) with $s \leq \lambda_{1}-\delta$ that satisfies (a)-(b) with $\delta$ replaced by $\gamma$.

Similarly, since $\mathcal{E}_{n}$ is normally attracting, there is no solution $(u(\xi), w(\xi), s)$ of the system (5.4)-(5.6) with $\lambda_{n}+\delta \leq s$ that satisfies (1).

Letting $\epsilon=\min \left(\delta, \gamma, \epsilon_{1}, \ldots, \epsilon_{n}\right)$, the result is proved. 


\section{Invertible Difusion Matrix}

In this section we consider the convection-diffusion system (1.1) with $B\left(u_{-}\right)$invertible. In the interest of generality, we do not assume that $A\left(u_{-}\right)$is strictly hyperbolic.

Theorem 6.1. In Eq. (1.1) assume:

(1) $A(u)$ and $B(u)$ are $C^{2}$ functions of $u$.

(2) $A\left(u_{-}\right)$has a simple real eigenvalue $\lambda_{1}$ with left eigenvector $\ell_{1}$, right eigenvector $r_{1}$, $\ell_{1} r_{1}=1$.

(3) $\ell_{1}\left(D A\left(u_{-}\right) r_{1}\right) r_{1}>0$.

(4) $B\left(u_{-}\right)$is invertible.

(5) $\ell_{1} B\left(u_{-}\right) r_{1}>0$.

(6) $B\left(u_{-}\right)^{-1}\left(A\left(u_{-}\right)-\lambda_{1} I\right)$ has no purely imaginary eigenvalues other than 0.

Let $a_{1}=\ell_{1}\left(D A\left(u_{-}\right) r_{1}\right) r_{1}>0$ and $b_{1}=\ell_{1} B\left(u_{-}\right) r_{1}>0$. Then for small $\sigma<0$ there exists $a$ traveling-wave solution $u(x-s t)$ of Eq. (1.1), $s=\lambda_{1}+\sigma$, with

$$
\begin{aligned}
u(-\infty) & =u_{-}, \\
u^{\prime}(-\infty) & =0, \\
u(+\infty) & =u_{-}+\frac{2 b_{1}}{a_{1}} \sigma r_{1}+o(\sigma), \\
u^{\prime}(+\infty) & =0,
\end{aligned}
$$

and

$$
u(\xi) \sim u_{-} \text {and } u^{\prime}(\xi) \sim 0 \text { for }-\infty<\xi<+\infty .
$$

Assumption (3) is genuine nonlinearity of one characteristic field. Assumptions (4)-(6) are the Majda-Pego conditions. This theorem is analagous to the Majda-Pego Theorem for conservation laws, but the proof is different. If assumption (5) is changed to $\ell_{1} B\left(u_{-}\right) r_{1}<0$, then the traveling waves are defined for $\sigma>0$; otherwise the conclusions of the theorem are unchanged.

The formula for $u(+\infty)$ gives a curve $u_{+}(s)$ of right states of traveling waves with left state $u_{-}$and speed $s$.

Proof. We make the substitutions (4.2)-(4.3), where $v$ and $\sigma$ satisfy Eqs. (4.4)-(4.7), in Eq. (1.1). We obtain

$$
\left(A\left(u_{-}+v\right)-\left(\lambda_{1}+\sigma\right) I\right) v^{\prime}=\left(B\left(u_{-}+v\right) v^{\prime}\right)^{\prime} .
$$

Let $C(v)=A\left(u_{-}+v\right)-\lambda_{1} I$ and $E(v)=B\left(u_{-}+v\right)$. Then Eq. (6.2) becomes

$$
(C(v)-\sigma I) v^{\prime}=\left(E(v) v^{\prime}\right)^{\prime}
$$

where:

$\left(1^{\prime}\right) C(v)$ and $E(v)$ are $C^{2}$ functions of $v$.

$\left(2^{\prime}\right) C(0)$ has a simple real eigenvalue 0 with left eigenvector $\ell_{1}$, right eigenvector $r_{1}, \ell_{1} r_{1}=1$.

$\left(3^{\prime}\right) \ell_{1}\left(D C(0) r_{1}\right) r_{1}=a_{1}>0$.

$\left(4^{\prime}\right) E(0)$ is invertible.

$\left(5^{\prime}\right) \ell_{1} E(0) r_{1}=b_{1}>0$.

$\left(6^{\prime}\right) E(0)^{-1} C(0)$ has no purely imaginary eigenvalues other than 0 . 
Let $E(v)=E(0) \tilde{E}(v)$, so that $\tilde{E}(0)=I$. Let

$$
w=\tilde{E}(v) v^{\prime}
$$

Then

$$
E(0) w^{\prime}=(E(0) w)^{\prime}=\left(E(0) \tilde{E}(v) v^{\prime}\right)^{\prime}=\left(E(v) v^{\prime}\right)^{\prime} .
$$

From Eqs. (6.3)-(6.5) we obtain the system

$$
\begin{aligned}
v^{\prime} & =\tilde{E}(v)^{-1} w \\
w^{\prime} & =E(0)^{-1}(C(v)-\sigma I) \tilde{E}(v)^{-1} w .
\end{aligned}
$$

We append the equation

$$
\sigma^{\prime}=0
$$

System (6.6)-(6.8) is a $C^{2}$ autonomous ODE on $\mathbb{R}^{2 n+1}$ with the $(n+1)$-dimensional plane of equilibria $w=0$. For $\sigma$ near 0 we want to find solutions $(v(\xi), w(\xi), \sigma)$ that satisfy (4.13)-(4.15). Such solutions must lie in the center manifold of $(v, w, \sigma)=(0,0,0)$.

By $\left(4^{\prime}\right)$ and $\left(5^{\prime}\right)$ we can choose a basis $S=\left(s_{1}, \ldots, s_{n}\right)$ for $\mathbb{R}^{n}$ such that

(a) $s_{1}=\frac{1}{b_{1}} r_{1}$, so $\ell_{1} E(0) s_{1}=1$.

(b) $\ell_{1} E(0) s_{i}=0$ for $i=2, \ldots, n$.

Make the change of variables $v=S x$ and $w=S y$. Then the system (6.6)-(6.8) becomes the $C^{2}$ system

$$
\begin{aligned}
x^{\prime} & =S^{-1} \tilde{E}(S x)^{-1} S y, \\
y^{\prime} & =S^{-1} E(0)^{-1}(C(S x)-\sigma I) \tilde{E}(S x)^{-1} S y, \\
\sigma^{\prime} & =0 .
\end{aligned}
$$

Since $\tilde{E}(0)=I$, the linearization of the system $(6.9)-(6.11)$ at the equilibrium $(x, y, \sigma)=$ $(0,0,0)$ is

$$
\begin{aligned}
x^{\prime} & =y, \\
y^{\prime} & =S^{-1} E(0)^{-1} C(0) S y, \\
\sigma^{\prime} & =0 .
\end{aligned}
$$

The characteristic polynomial of the linear system $(6.12)-(6.14)$ is $p(\lambda)=\lambda^{n+1} q(\lambda)$, where $q(\lambda)$ is the characteristic polynomial of $E(0)^{-1} C(0)$. By $\left(2^{\prime}\right), 0$ is an eigenvalue of $E(0)^{-1} C(0)$ with one-dimensional eigenspace spanned by $r_{1}$. Moreover, $E(0)^{-1} C(0)$ has no generalized eigenvectors for the eigenvalue 0 . To see this, suppose there is a vector $v$ such that $E(0)^{-1} C(0) v=r_{1}$. Then $C(0) v=E(0) r_{1}$. Multiplication by $\ell_{1}$ yields $0=\ell_{1} E(0) r_{1}$, which contradicts $\left(5^{\prime}\right)$. Thus $q(\lambda)$ has a simple root $\lambda=0$, and by $\left(6^{\prime}\right)$ there are no other pure imaginary roots. Thus the center subspace of the linear system $(6.12)-(6.14)$ is the $(n+2)$ dimensional generalized eigenspace for the eigenvalue 0 , which is $x y_{1} \sigma$-space. Since all points $(x, 0, \sigma)$ are equilibria of the system $(6.6)-(6.8)$, as in Sec. 4 the equations for the center manifold are (4.23) with each $h_{i}$ a $C^{1}$ function such that $y_{1} h_{i}$ is $C^{2}$, and $h_{i}(0,0,0)=0$.

The differential equation (6.9)-(6.11), restricted to the center manifold, is obtained as in Sec. 4. Note that to obtain $y_{1}^{\prime}$, we simply replace $S^{-1} E(0)^{-1}=(E(0) S)^{-1}$ in the right hand 
side of Eq. (6.13) by its first row; by (a) and (b), this is $\ell_{1}$. Thus we obtain the $C^{2}$ system

$$
\begin{aligned}
x_{1}^{\prime} & =y_{1}, \\
x_{2}^{\prime} & =y_{1} h_{2}, \\
\vdots & \\
x_{n}^{\prime} & =y_{1} h_{n}, \\
y_{1}^{\prime} & =\ell_{1}(C(S x)-\sigma I) \tilde{E}(S x)^{-1} S\left(y_{1}, y_{1} h_{2}, \ldots, y_{1} h_{n}\right)^{\top} \\
\sigma^{\prime} & =0 .
\end{aligned}
$$

Using $\ell_{1} C(0)=0$ and $h_{i}(0,0,0)=0$, Eq. (6.18) can be expanded as follows:

$$
\begin{aligned}
y_{1}^{\prime} & =y_{1} \ell_{1}(C(0)+D C(0) S x+\ldots-\sigma I)(I+\ldots) S\left(1, h_{2}, \ldots, h_{n}\right)^{\top} \\
& =y_{1} \ell_{1}(D C(0) S x+\ldots-\sigma I)(I+\ldots) S\left(1, h_{2}, \ldots, h_{n}\right)^{\top} \\
& =y_{1} \ell_{1}\left\{(D C(0) S x-\sigma I) S(1,0, \ldots, 0)^{\top}+\ldots\right\} \\
& =y_{1} \ell_{1}\left\{\left(\sum_{i=1}^{n} x_{i} D C(0) s_{i}-\sigma I\right) s_{1}+\ldots\right\} \\
& =y_{1}\left(\sum_{i=1}^{n} c_{i} x_{i}-\frac{1}{b_{1}} \sigma+\ldots\right),
\end{aligned}
$$

where $c_{i}=\ell_{1}\left(D C(0) s_{i}\right) s_{1}$. In particular, $c_{1}=\frac{a_{1}}{b_{1}^{2}} \neq 0$.

Since $a_{1}>0$ and $b_{1}>0$, the remainder of the proof is similar to that of Theorem 4.1.

\section{Degenerate Diffusion}

In this section, following Sainsaulieu, we consider the convection-diffusion system (1.1) and assume that the first $p$ equations contain no diffusion terms. Thus we write $u=\left(u^{1}, u^{2}\right)$ with $u^{1} \in \mathbb{R}^{p}, u^{2} \in \mathbb{R}^{q}$, and $p+q=n$;

$$
A(u)=\left(\begin{array}{ll}
A_{1}(u) & A_{2}(u) \\
A_{3}(u) & A_{4}(u)
\end{array}\right)
$$

with $A_{1}$ a $p \times p$ matrix, $A_{2}$ a $p \times q$ matrix, $A_{3}$ a $q \times p$ matrix, and $A_{4}$ a $q \times q$ matrix; and

$$
B(u)=\left(\begin{array}{cc}
0 & 0 \\
B_{1}(u) & B_{2}(u)
\end{array}\right)
$$

with $B_{1}$ a $q \times p$ matrix and $B_{2}$ a $q \times q$ matrix. As in Sec. 6 , in the interest of generality we do not assume that $A\left(u_{-}\right)$is strictly hyperbolic.

Theorem 7.1. In Eq. (1.1) with the above assumptions and notation, assume:

(1) $A(u)$ and $B(u)$ are $C^{2}$ functions of $u$.

(2) $A\left(u_{-}\right)$has a simple real eigenvalue $\lambda$ with left eigenvector $\ell$, right eigenvector $r, \ell r=1$.

(3) $A_{1}\left(u_{-}\right)-\lambda I$ is invertible.

(4) $\ell\left(D A\left(u_{-}\right) r\right) r>0$.

(5) $-B_{1}\left(u_{-}\right)\left(A_{1}\left(u_{-}\right)-\lambda I\right)^{-1} A_{2}\left(u_{-}\right)+B_{2}\left(u_{-}\right)$is invertible.

(6) $\ell B\left(u_{-}\right) r>0$.

(7) For each nonzero real $\omega$, the equation $\left(A\left(u_{-}\right)-\lambda I\right) v=i \omega B\left(u_{-}\right) v$ has only the trivial solution. 
Let $a=\ell\left(D A\left(u_{-}\right) r\right) r>0$ and $b=\ell B\left(u_{-}\right) r>0$. Then for small $\sigma<0$ there exists $a$ traveling-wave solution $u(x-s t)$ of Eq. (4.1), $s=\lambda+\sigma$, with

$$
\begin{aligned}
u(-\infty) & =u_{-}, \\
u^{\prime}(-\infty) & =0, \\
u(+\infty) & =u_{-}+\frac{2 b}{a} \sigma r+o(\sigma), \\
u^{\prime}(+\infty) & =0,
\end{aligned}
$$

and

$$
u(\xi) \sim u_{-} \text {and } u^{\prime}(\xi) \sim 0 \text { for }-\infty<\xi<+\infty .
$$

Assumption (4) is genuine nonlinearity of one characteristic field. Assumptions (5)-(7) are analagous to assumptions (4)-(6) of Theorem 6.1. If assumption (6) is changed to $\ell B\left(u_{-}\right) r<0$, then the traveling waves are defined for $\sigma>0$; otherwise the conclusions of the theorem are unchanged. Assumption (3) is special to the degenerate diffusion case.

The formula for $u(+\infty)$ gives a curve $u_{+}(s)$ of right states of traveling waves with left state $u_{-}$and speed $s$.

Proof. Substituting $u=u(x-s t)$ into Eq. (1.1) yields

$$
\begin{aligned}
& \left(A_{1}(u)-s I\right) u^{1 \prime}+A_{2}(u) u^{2 \prime}=0, \\
& A_{3}(u) u^{1 \prime}+\left(A_{4}(u)-s I\right) u^{2 \prime}=\left(B_{1}(u) u^{1 \prime}+B_{2}(u) u^{2 \prime}\right)^{\prime} .
\end{aligned}
$$

For $(u, s)$ near $\left(u_{-}, \lambda\right)$ we can invert $A_{1}(u)-s I$ by (3). Thus Eq. (7.3) can be rewritten

$$
u^{1 \prime}=-\left(A_{1}(u)-s I\right)^{-1} A_{2}(u) u^{2 \prime} .
$$

Substituting (7.5) into Eq. (7.4) yields

$$
\begin{aligned}
& \left\{-A_{3}(u)\left(A_{1}(u)-s I\right)^{-1} A_{2}(u)+A_{4}(u)-s I\right\} u^{2 \prime}= \\
& \left\{\left(-B_{1}(u)\left(A_{1}(u)-s I\right)^{-1} A_{2}(u)+B_{2}(u)\right) u^{2 \prime}\right\}^{\prime} .
\end{aligned}
$$

In Eqs. (7.5)-(7.6), let $u(\xi)=u_{-}+v(\xi)$ and $s=\lambda+\sigma$, where $v$ and $\sigma$ satisfy Eqs. (4.4)(4.7). We obtain the $C^{2}$ system

$$
\begin{aligned}
v^{1 \prime} & =G(v, \sigma) v^{2 \prime}, \\
C(v, \sigma) v^{2 \prime} & =\left(E(v, \sigma) v^{2 \prime}\right)^{\prime},
\end{aligned}
$$

with

$$
\begin{aligned}
& G(v, \sigma)=-\left\{A_{1}\left(u_{-}+v\right)-(\lambda+\sigma) I\right\}^{-1} A_{2}\left(u_{-}+v\right), \\
& C(v, \sigma)=-A_{3}\left(u_{-}+v\right)\left\{A_{1}\left(u_{-}+v\right)-(\lambda+\sigma) I\right\}^{-1} A_{2}\left(u_{-}+v\right)+A_{4}\left(u_{-}+v\right)-(\lambda+\sigma) I, \\
& E(v, \sigma)=-B_{1}\left(u_{-}+v\right)\left\{A_{1}\left(u_{-}+v\right)-(\lambda+\sigma) I\right\}^{-1} A_{2}\left(u_{-}+v\right)+B_{2}\left(u_{-}+v\right) .
\end{aligned}
$$

Notice $E(0,0$ is invertible by assumption (5). Let $E(v, \sigma)=E(0,0) \tilde{E}(v, \sigma)$, so that $\tilde{E}(0,0)=$ I. Let

$$
w^{2}=\tilde{E}(v, \sigma) v^{2 \prime}
$$

Then

$$
E(0,0) w^{2 \prime}=\left(E(0,0) w^{2}\right)^{\prime}=\left(E(0,0) \tilde{E}(v, \sigma) v^{2 \prime}\right)^{\prime}=\left(E(v, \sigma) v^{2 \prime}\right)^{\prime}
$$


From Eqs. (7.7)-(7.10) we obtain the system

$$
\begin{aligned}
v^{1 \prime} & =G(v, \sigma) \tilde{E}(v, \sigma)^{-1} w^{2} \\
v^{2 \prime} & =\tilde{E}(v, \sigma)^{-1} w^{2} \\
w^{2 \prime} & =E(0,0)^{-1} C(v, \sigma) \tilde{E}(v, \sigma)^{-1} w^{2} .
\end{aligned}
$$

We append the equation

$$
\sigma^{\prime}=0 .
$$

System (7.11)-(7.14) is a $C^{2}$ autonomous ODE on $\mathbb{R}^{n+q+1}$ with the $(n+1)$-dimensional plane of equilibria $w^{2}=0$. We want to find solutions $\left(v(\xi), w^{2}(\xi), \sigma\right)$ that stay near the origin for all time and satisfy

$$
\begin{aligned}
& v(-\infty)=0, \quad w^{2}(-\infty)=0, \\
& v(+\infty) \sim 0, \quad w^{2}(+\infty)=0 .
\end{aligned}
$$

Such solutions must lie in the center manifold of $\left(v, w^{2}, \sigma\right)=(0,0,0)$.

We collect some facts about $\mathrm{C}(0,0)$ and $\mathrm{E}(0,0)$. They are analogs of assumptions (2), (4), (6), and (7).

Lemma 7.2. $C(0,0)$ has an eigenvalue 0 with one-dimensional eigenspace. A left eigenvector is $\ell^{2}$ and a right eigenvector is $r^{2}$.

Proof. We write the equation

$$
\left(A\left(u_{-}\right)-\lambda I\right) v=0
$$

as the system

$$
\begin{aligned}
& \left(A_{1}\left(u_{-}\right)-\lambda I\right) v^{1}+A_{2}\left(u_{-}\right) v^{2}=0, \\
& A_{3}\left(u_{-}\right) v^{1}+\left(A_{4}\left(u_{-}\right)-\lambda I\right) v^{2}=0 .
\end{aligned}
$$

Since $A_{1}\left(u_{-}\right)-\lambda I$ is invertible by (3), we can solve Eq. (7.16) for $v^{1}$ and substitute the answer into Eq. (7.17). We find that $v=\left(v^{1}, v^{2}\right)$ is a solution of Eq. (7.15) if and only if

$$
\begin{aligned}
v^{1} & =-\left(A_{1}\left(u_{-}\right)-\lambda I\right)^{-1} A_{2}\left(u_{-}\right) v^{2}, \\
C(0,0) v^{2} & =0 .
\end{aligned}
$$

It then follows from (2) that the only solutions of Eq. (7.19) are multiples of $r^{2}$.

Similarly, we write the equation

$$
z\left(A\left(u_{-}\right)-\lambda I\right)=0
$$

as the system

$$
\begin{aligned}
& z^{1}\left(A_{1}\left(u_{-}\right)-\lambda I\right)+z^{2} A_{3}\left(u_{-}\right)=0, \\
& z^{1} A_{2}\left(u_{-}\right)+z^{2}\left(A_{4}\left(u_{-}\right)-\lambda I\right)=0 .
\end{aligned}
$$

Thus $z=\left(z^{1}, z^{2}\right)$ is a solution of Eq. (7.20) if and only if

$$
\begin{aligned}
z^{1} & =-z^{2} A_{3}\left(u_{-}\right)\left(A_{1}\left(u_{-}\right)-\lambda I\right)^{-1}, \\
z^{2} C(0,0) & =0 .
\end{aligned}
$$

It then follows from (2) that the only solutions of Eq. (7.22) are multiples of $\ell_{2}$. 
Lemma 7.3. $\ell^{2}\left(D_{1} C(0,0) r\right) r^{2}=a>0$.

Proof. From Eq. (7.18),

$$
r^{1}=-\left(A_{1}\left(u_{-}\right)-\lambda I\right)^{-1} A_{2}\left(u_{-}\right) r^{2}
$$

From Eq. (7.21),

$$
\ell^{1}=-\ell^{2} A_{3}\left(u_{-}\right)\left(A_{1}\left(u_{-}\right)-\lambda I\right)^{-1}
$$

Now

$$
a=\ell\left(D A\left(u_{-}\right) r\right) r=\left(\begin{array}{ll}
\ell^{1} & \ell^{2}
\end{array}\right)\left(\begin{array}{ll}
D A_{1}\left(u_{-}\right) r & D A_{2}\left(u_{-}\right) r \\
D A_{3}\left(u_{-}\right) r & D A_{4}\left(u_{-}\right) r
\end{array}\right)\left(\begin{array}{l}
r^{1} \\
r^{2}
\end{array}\right)
$$

Substitute Eq. (7.23) and Eq. (7.24) into Eq. (7.25), multiply out, and compare to

$$
\begin{aligned}
\ell^{2}\left(D_{1} C(0,0) r\right) & r^{2}=\ell^{2}\left\{-D A_{3}\left(u_{1}\right) r\left(A_{1}\left(u_{-}\right)-\lambda I\right)^{-1} A_{2}\left(u_{-}\right)\right. \\
+ & A_{3}\left(u_{-}\right)\left(A_{1}\left(u_{-}\right)-\lambda I\right)^{-1} D A_{1}\left(u_{-}\right) r\left(A_{1}\left(u_{-}\right)-\lambda I\right)^{-1} A_{2}\left(u_{-}\right) \\
& \left.-A_{3}\left(u_{-}\right)\left(A_{1}\left(u_{-}\right)-\lambda I\right)^{-1} D A_{2}\left(u_{-}\right) r+D A_{4}\left(u_{1}\right) r\right\} r^{2} .
\end{aligned}
$$

Lemma 7.4. $\ell^{2} E(0,0) r^{2}=b>0$.

Proof. From (6) we have

$$
\left(\begin{array}{ll}
\ell^{1} & \ell^{2}
\end{array}\right)\left(\begin{array}{cc}
0 & 0 \\
B_{1}\left(u_{-}\right) & B_{2}\left(u_{-}\right)
\end{array}\right)\left(\begin{array}{l}
r^{1} \\
r^{2}
\end{array}\right)=b>0
$$

i.e.,

$$
\ell^{2}\left(B_{1}\left(u_{-}\right) r^{1}+B_{2}\left(u_{-}\right) r^{2}\right)=b>0 .
$$

Substituting (7.23) into (7.27) yields the result.

Lemma 7.5. $E(0,0)^{-1} C(0,0)$ has no purely imaginary eigenvalues other than 0 .

Proof. Suppose there is a nonzero vector $v^{2}$ such that $E(0,0)^{-1} C(0,0) v^{2}=i \omega v^{2}$ with $\omega$ a nonzero real number. Then $C(0,0) v^{2}=i \omega E(0,0) v^{2}$, i.e.,

$$
\begin{aligned}
\left\{-A_{3}\left(u_{-}\right)\left(A_{1}\left(u_{-}\right)-\lambda I\right)^{-1} A_{2}\left(u_{-}\right)+\right. & \left.A_{4}\left(u_{-}\right)-\lambda I\right\} v^{2}= \\
& i \omega\left\{-\left(B_{1}\left(u_{-}\right)\left(A_{1}\left(u_{-}\right)-\lambda I\right)^{-1} A_{2}\left(u_{-}\right)+B_{2}\left(u_{-}\right)\right\} v^{2} .\right.
\end{aligned}
$$

Letting $v^{1}=-\left(A_{1}\left(u_{-}\right)-\lambda I\right)^{-1} A_{2}\left(u_{-}\right) v^{2}$, we find that

$$
\left(\begin{array}{cc}
A_{1}\left(u_{-}\right)-\lambda I & A_{2}\left(u_{-}\right) \\
A_{3}\left(u_{-}\right) & A_{4}\left(u_{-}\right)-\lambda I
\end{array}\right)\left(\begin{array}{l}
v^{1} \\
v^{2}
\end{array}\right)=i \omega\left(\begin{array}{cc}
0 & 0 \\
B_{1}\left(u_{-}\right) & B_{2}\left(u_{-}\right)
\end{array}\right)\left(\begin{array}{l}
v^{1} \\
v^{2}
\end{array}\right) .
$$

This contradicts $(7)$.

Let $R=\left(r_{1}, \ldots, r_{n}\right)$ be a basis for $\mathbb{R}^{n}$ such that $r_{1}=\frac{1}{b} r$ and $\ell r_{i}=0$ for $i=2, \ldots, n$. Then the first row of $R^{-1}$ is $b \ell$.

Let $S=\left(s_{1}, \ldots, s_{q}\right)$ be a basis for $\mathbb{R}^{q}$ such that

(a) $s_{1}=\frac{1}{b} r^{2}$, so $\ell^{2} E(0,0) s_{1}=1$.

(b) $\ell^{2} E(0,0) s_{i}=0$ for $i=2, \ldots, q$. 
We can choose such a basis by (5) and Lemma 7.4.

Make the change of variables $v=R x$ and $w_{2}=S y$. Then the system $(7.11)-(7.14)$ becomes

$$
\begin{aligned}
x^{\prime} & =R^{-1}\left(\begin{array}{c}
G(R x, \sigma) \\
I
\end{array}\right) \tilde{E}(R x, \sigma)^{-1} S y \\
y^{\prime} & =S^{-1} E(0,0)^{-1} C(R x, \sigma) \tilde{E}(R x, \sigma)^{-1} S y, \\
\sigma^{\prime} & =0 .
\end{aligned}
$$

The linearization of the system (7.30)-(7.32) at the equilibrium $(x, y, \sigma)=(0,0,0)$ is

$$
\begin{aligned}
x^{\prime} & =R^{-1}\left(\begin{array}{c}
G(0,0) \\
I
\end{array}\right) S y, \\
y^{\prime} & =S^{-1} E(0,0)^{-1} C(0,0) S y, \\
\sigma^{\prime} & =0 .
\end{aligned}
$$

The characteristic polynomial of the linear system (7.33)-(7.35) is $p(\lambda)=\lambda^{n+1} q(\lambda)$, where $q(\lambda)$ is the characteristic polynomial of $E(0,0)^{-1} C(0,0)$. By Lemma 7.2, 0 is an eigenvalue of $E(0,0)^{-1} C(0,0)$ with one-dimensional eigenspace spanned by $r^{2}$. Moreover, $E(0,0)^{-1} C(0,0)$ has no generalized eigenvectors for the eigenvalue 0 . For suppose there is a vector $v^{2}$ such that $E(0,0)^{-1} C(0,0) v^{2}=r^{2}$. Then $C(0,0) v^{2}=E(0,0) r^{2}$. By Lemma 7.2, multiplication by $\ell^{2}$ yields $0=\ell^{2} E(0,0) r^{2}$, which contradicts Lemma 7.4. Thus $q(\lambda)$ has a simple root $\lambda=0$, and by Lemma 7.5 there are no other pure imaginary roots. Hence the center subspace of the linear system $(7.33)-(7.35)$ is the $(n+2)$-dimensional generalized eigenspace for the eigenvalue 0, which is $x y_{1} \sigma$-space. As in Secs. 4 and 6 , we find that the equations for the center manifold are

$$
y_{i}=y_{1} h_{i}\left(x, y_{1}, \sigma\right), \quad i=2, \ldots, q,
$$

with each $h_{i}$ a $C^{1}$ function such that $y_{1} h_{i}$ is $C^{2}$, and $h_{i}(0,0,0)=0$.

The differential equation (7.30)-(7.32), restricted to the center manifold, is the $C^{2}$ system

$$
\begin{aligned}
x^{\prime} & =R^{-1}\left(\begin{array}{c}
G(R x, \sigma) \\
I
\end{array}\right) \tilde{E}(R x, \sigma)^{-1} S\left(y_{1}, y_{1} h_{2}, \ldots, y_{1} h_{q}\right)^{\top}, \\
y_{1}^{\prime} & =\ell^{2} C(R x, \sigma) \tilde{E}(R x, \sigma)^{-1} S\left(y_{1}, y_{1} h_{2}, \ldots, y_{1} h_{q}\right)^{\top}, \\
\sigma^{\prime} & =0 .
\end{aligned}
$$

Using $h_{i}(0,0,0)=0$, Eq. (7.23) and our knowledge of $R^{-1}$, Eq. (7.37) can be expanded as follows:

$$
\begin{aligned}
x^{\prime} & =y_{1}\left(R^{-1}\left(\begin{array}{c}
G(0,0) \\
I
\end{array}\right) S(1,0, \ldots, 0)+\ldots\right) \\
& =y_{1}\left(R^{-1}\left(\begin{array}{c}
G(0,0) \\
I
\end{array}\right) s_{1}+\ldots\right) \\
& =\frac{1}{b} y_{1}\left(R^{-1}\left(\begin{array}{c}
r^{1} \\
r^{2}
\end{array}\right)+\ldots\right) \\
& =y_{1}\left\{(1,0, \ldots, 0)^{\top}+\ldots\right\}
\end{aligned}
$$


Using $\ell^{2} C(0,0)=0$ and $h_{i}(0,0,0)=0$, Eq. (7.38) can be expanded as follows:

$$
\begin{aligned}
y_{1}^{\prime} & =y_{1} \ell^{2}\left(C(0,0)+D_{1} C(0,0) R x+D_{2} C(0,0) \sigma+\ldots\right)(I+\ldots) S\left(1, h_{2}, \ldots, h_{q}\right)^{\top} \\
& =y_{1} \ell^{2}\left(D_{1} C(0,0) R x+D_{2} C(0,0) \sigma+\ldots\right)(I+\ldots) S\left(1, h_{2}, \ldots, h_{q}\right)^{\top} \\
& =y_{1} \ell^{2}\left\{\left(D_{1} C(0,0) R x+D_{2} C(0,0) \sigma\right) s_{1}+\ldots\right\} \\
& =y_{1} \ell^{2}\left\{\left(D_{1} C(0,0)\left(x_{1} r_{1}+\ldots+x_{n} r_{n}\right)+D_{2} C(0,0) \sigma\right) s_{1}+\ldots\right\} \\
& =y_{1}\left(\sum_{i=1}^{n} c_{i} x_{i}+e \sigma+\ldots\right),
\end{aligned}
$$

where $c_{i}=\ell^{2}\left(D_{1} C(0,0) r_{i}\right) s_{1}$ and $e=\ell^{2} D_{2} C(0,0) s_{1}$. have

By Lemma 7.3, $c_{1}=\frac{a}{b^{2}}$. Moreover, $e=-\frac{1}{b}$, because using Eq. (7.24) and Eq. (7.23) we

$$
\ell^{2} D_{2} C(0,0) r^{2}=\ell^{2}\left(-A_{3}\left(u_{-}\right)\left(A_{1}\left(u_{-}\right)-\lambda I\right)^{-2} A_{2}\left(u_{-}\right)-I\right) r^{2}=\ell^{1}\left(-r^{1}\right)-\ell^{2} r^{2}=-\ell r=-1 .
$$

Since $a_{1}>0$ and $b_{1}>0$, the remainder of the proof is similar to that of Theorem 4.1.

\section{Discussion}

The reader may wish to compare the hypotheses of our Theorem 7.1 and its analog in [11], Sainsaulieu's Theorem 2.1. Sainsaulieu explicitly assumes our assumptions (1)-(4) and (6). In addition, Sainsaulieu assumes

$$
\text { ker } B\left(u_{-}\right) \cap\left(A\left(u_{-}\right)-\lambda I\right)^{-1} \text { range } B\left(u^{-}\right)=\{0\} .
$$

This assumption is clearly misstated, since $A\left(u_{-}\right)-\lambda I$ is not invertible. However, Sainsaulieu uses this assumption to derive our assumption (5) in his Lemma 4.2; it is this lemma that is then used in Sainsaulieu's proof. Sainsaulieu does not state our assumption (7) or any analog of it. However, his proof actually requires this assumption (toward the bottom of $\mathrm{p}$. 1296 of [11], where he says that a certain ODE has a unique bounded solution).

To generalize our uniqueness result Theorem 5.1 to the case in which $B\left(u_{-}\right)$is invertible, one must confront the possibility that $B\left(u_{-}\right)^{-1}\left(A\left(u_{-}\right)-s I\right)$ has pure imaginary eigenvalues at some $s$ that is not an eigenvalue of $A\left(u_{-}\right)$. Sainsaulieu's uniqueness proof in Sec. 5 of [11] appears not to take this possibility into account.

\section{ACKNOWLEDGMENTS}

I thank Xiao-Biao Lin for introducing me to convection-diffusion equations that are not in conservation form, and Michael Shearer for showing me Sainsaulieu's paper [11].

\section{REFERENCES}

[1] S. Čanić, On the influence of viscosity on Riemann solutions, J. Dyn. Diff. Eqns. 9 (1997), 977-998.

[2] G. Dal Maso, P. G. LeFloch, and F. Murat, Definition and weak stability of nonconservative products, J. Math. Pures Appl. 74 (1995), 483-548.

[3] J. Guckenheimer and P. Holmes, "Nonlinear Oscillations, Dynamical Systems, and Bifurcations of Vector Fields," Springer-Verlag, New York, 1983.

[4] P. D. Lax, "Hyperbolic Systems of Conservation Laws and the Mathematical Theory of Shock Waves," SIAM, Philadelphia, 1972.

[5] N. Fenichel, Geometric Singular Perturbation Theory for Ordinary Differential Equations, J. Differential Eqs. 31 (1979), 53-98.

[6] P. G. LeFloch, Shock waves for nonlinear hyperbolic systems in nonconservative form, preprint 593, Institute for Mathematics and its Applications, University of Minnesota, Minneapolis, 1989. 
[7] P. G. LeFloch and T.-P. Liu, Existence theory for nonlinear hyperbolic systems in nonconsevative form, Forum Math. 5 (1993), 261-280.

[8] P. G. LeFloch and A. E. Tzavaras, Existence theory for the Riemann problem for non-conservative hyperbolic systems, C. R. Acad. Sci. Paris 323, Série I, 347-352.

[9] A. Majda and R. Pego, Stable viscosity matrices for systems of conservation laws, J. Differential Eqs. 56 (1985), 229-262.

[10] P.-A. Raviart and L. Sainsaulieu, A nonconservative hyperbolic system modeling spray dynamics. I. Solution of the Riemann Problem, Math. Models Methods Appl. Sci. 5 (1995), 297-333.

[11] L. Sainsaulieu, Traveling-wave solutions of convection-diffusion systems in nonconservation form, SIAM J. Math. Anal. 27 (1996), 1286-1310.

[12] J. Smoller, "Shock Waves and Reaction-Diffusion Equations," Springer-Verlag, New York, 1983.

[13] H. B. Stewart and B. Wendroff, Two-phase flow: models and methods, J. Comput. Phys. 56 (1984), 363-409.

[14] J. A. Trangenstein and P. Colella, A higher-order Godunov method for modeling finite deformation in elastic-plastic solids, Comm. Pure Appl. Math. 44 (1991), 41-100.

[15] A. Vanderbauwhede and S. A. van Gils, Center manifolds and contractions on a scale of Banach spaces, J. Funct. Anal. 72 (1987), 209-224.

Mathematics Department, North Carolina State University, Raleigh, NC 27695

E-mail address: schecter@math.ncsu.edu 